\title{
Aportaciones y diferencias entre comunicación en salud, comunicación para el desarrollo y para el cambio social
}

\author{
Carlos Andrés Martínez Beleño \\ María Silvina Sosa Gómez \\ Universidad Autónoma del Caribe (Colombia)
}

\section{Palabras clave}

Comunicación en Salud, Comunicación para el Desarrollo,

Comunicación para el Cambio Social,

Salud

\begin{abstract}
Resumen
Este artículo pretende reflexionar y asimismo, poner en discusión las perspectivas y miradas de los investigadores de la comunicación en salud, para el desarrollo y para el cambio social. En ese sentido, serán los postulados de Ríos, Gumucio, Atoche, Vega y Mosquera quienes aludirán sobre los aportes teóricos en las áreas de la comunicación, que en últimas generan espacio de diálogo para lograr un cambio en las comunidades donde se implementa estrategias de comunicación y salud.

Se pretende entonces, vislumbrar el panorama desde el soporte teórico de cómo se trabaja y en función de que se investiga en comunicación y las áreas mencionadas. Si bien este texto presenta las miradas de los estudiosos de sobre algunos abordajes de la comunicación, los autores presentaran a su vez la importancia de este tipo de comunicación y su implementación en el bienestar social por ejemplo.

Lo que busca este trabajo, es percibir como un área interviene en otras o del mismo modo una sirve como estrategia para lograr las intenciones de otras. Es así como la Comunicación en salud, para el desarrollo y para el cambio social se correlacionan y confluyen entre sí, en busca de generar cambios no solo de comportamientos sino, de estilos de vida saludables para las poblaciones donde se está haciendo intervención. Mirado así, cuando se alude a las diferencias, se presentan miradas distintas pero complementarias y acciones concretas para públicos divergentes. De este modo, lo más interesante es tener presente que éstas sustancialmente buscan un cambio comportamiento en función del contexto social.
\end{abstract}




\section{INTRODUCCIÓN}

Desde el transcurrir de una investigación en curso, surgió el siguiente interrogante: ¿En qué se diferencian la comunicación para el desarrollo de la comunicación para el cambio social y la Comunicación en salud?

Se pretenderá en este texto, aludir desde el soporte teórico a estos tres abordajes a fin de reflexionar y distinguir que aporta cada una y que las diferencian.

Para investigadores y estudiosos de estas disciplinas de la Comunicación, como lo son Tufte y Gumucio, la primera basa sus intenciones en teorías psicosociales y de persuasión así, como de relaciones públicas centrándose en cambios de comportamiento individuales gracias a la difusión de mensajes a públicos con características convergentes.

Asimismo, la segunda pretende lograr que la gente sea actante de los mismos procesos; que pueda proponerlos, formularlos, gestionarlos, evaluarlos y solucionarlos.

Y la última abarca el estudio y el uso de estrategias de comunicación para informar e influenciar decisiones individuales y comunitarias que mejoren la salud pública y personal. (Atoche, 2003)

\section{COMUNICACIÓN EN SALUD, COMUNICACIÓN PARA EL DESARROLLO Y PARA EL CAMBIO SOCIAL}

Cuando se habla de comunicación se debe hacer una recapitulación para identificar el panorama actual y percibir su evolución histórica y social. La idea de que los países en proceso de desarrollo deberían emerger a partir de experiencias de los países desarrollados, dispuestos a compartir sus conocimientos y tecnologías a los más pobres, llevó a desarrollar un concepto de comunicación como una información asistencialista y difusionista durante los años cincuenta. Sin embargo, esta información no buscaba el diálogo con las poblaciones; la comunicación en éste período era un instrumento de transferencia de ideas y principalmente tecnología, con la justificación de que los países desarrollados sabían lo que convenía a los países subdesarrollados. Apoyaron la expansión de mercados y la incorporación al consumo de grandes masas de poblaciones marginales a través de mecanismos de persuasión y estrategias de transferencia de información y difusión de informaciones tecnológicas modelos verticales generados en laboratorios de empresas privadas, agencias de publicidad y universidades de Estados Unidos. (Sosa, 2010)

Bajo lo anterior, Rodríguez, Obregón \& Vega (2002) puntualizan que los medios masivos de comunicación se basan en la eficacia: tecnologías diseñadas para llegarle al mayor número de gente posible en el menor tiempo y con el menor esfuerzo. Mientras la publicidad y los medios comerciales tienen muy claro que construyen mensajes exclusivamente para vender, los mensajes generados por organizaciones y movimientos sociales tiene un objetivo muy diferente: el cambio social, la transformación de valores culturales, de creencias, de relaciones de poder.

Vistos estos planteamientos, es preciso mencionar que en los sesenta, empiezan a surgir las teorías de la dependencia, donde los organismos de cooperación internacional, como la Organización para la Educación, la Ciencia y la Cultura de las Naciones Unidas, UNESCO, y la Organización de las Naciones Unidas para la Agricultura y la Alimentación, FAO, defienden el derecho a la información y la adopción de estrategias de comunicación para el desarrollo; El desarrollo, era concebido como la necesidad de modelar a las naciones pobres a imagen y semejanza de los países industrializados; para lograr el salto 
los pueblos del tercer mundo debían despojarse de creencias, tradiciones y prácticas culturales que son un freno para la modernización.

Los modelos emergentes de las experiencias independentistas de África, Asia y América Latina ofrece una de las premisas principales explicando que las causas del subdesarrollo son estructurales y tienen que ver con la tenencia de la tierra, con la falta de libertades colectivas, con la opresión de las culturas indígenas, con la injusticia social y otros temas políticos y sociales y no solamente con la carencia de información y conocimiento. (Gumucio; 2007: 2).

Hacia los setentas se presentaron algunas tramas históricas, sociales, culturales, políticas ideológicas como fueron las luchas de distintos movimientos sociales en Europa, en los Estados Unidos, en América Latina, en cada espacio-tiempo con sus características propias. La lucha contra la discriminación sexual, racial y cultural, de clase, la lucha en defensa del medio ambiente, los golpes de Estado, con su nueva cara en América Latina y sus gobiernos militares que se arrastraban desde la década anterior las guerrillas en América Latina, las comunidades de base, los movimientos de liberación en África, la independencia de las ex colonias portuguesas, la lucha en Namibia, Amílcar, Cabral, julios, Nyerere, su liderazgo en África y su repercusión fuera de ellas, la China de Mao, La revolución cultural, las luchas político-sindicales y pedagógico . sindicales principalmente en Italia, la guerra de Vietnam, la lucha por los derechos civiles y el desbordamiento del clima político cultural, sectores sociales representativos por democratizar la información, como son la actuación de los campesinos, indígenas, obreros, mujeres, artistas, intelectuales, asociaciones cristianas de base y más actualmente de movimientos sociales de derechos humanos.

Se empieza entonces, a tratar y utilizar la comunicación no sólo como una finalidad publicitaria sino también como un instrumento de uso público, de todos, en una perspectiva de mayor diálogo, entendida como un derecho de todos, que valora la cultura local y la educación; Estos modelos promueven cambios sociales colectivos antes que individuales y acciones de comunicación desde las comunidades y no para las comunidades.

En últimas y en función de todo el panorama presentando, la comunicación es de dos, es de grupos, de sistemas, de dinámicas, de todo un contexto social y por ello hemos de tener en cuenta con quien estamos hablando, tenemos que considerar el universo cultural de las personas a quienes queremos llegar. Hemos de tener claro que el mensaje que vamos a emitir pueda ser recibido y admitido por la gente. (Rodríguez, Obregón, Vega: 2002: 16, 17).

Por una lado, la Comunicación para el Desarrollo surge de un proceso estratégico adoptado por agencias de cooperación internacional en la década de los 50 , en los Estados Unidos la impulsó la United States Agency for International Development (USAID) y en las Naciones Unidas FNUAP a través de campañas de comportamiento para temas de nutrición y desarrollo humano. A su vez, FAO empleó la educación para la formación de agricultores y tecnologías agrícolas saludables y UNICEF para el bienestar de los niños y sus madres. Asimismo, la comunicación para el desarrollo basa sus intenciones en teorías psicosociales y de persuasión como de relaciones públicas centrándose en cambios de comportamiento individuales gracias a la difusión de mensajes a públicos con características convergentes.

Para Martínez, (2012), la Comunicación para el Desarrollo busca establecer y diseñar los mecanismos apropiados al contexto local y hacer uso de los canales de comunicación 
existentes para visibilizar el logro y sostenibilidad de los proyectos, planes y programas que involucran al individuo de forma complejamente, en función del dialogo de doble vía entre los diferentes actores del proceso; permitiendo además, la implementación de herramientas comunicacionales individuales, grupales y de mesa con el fin de incidir favorablemente en las dinámicas sociales. Asimismo, se expresa, que además de actuar como agente movilizador, integra grupos humanos, desarrolla la actividad social, involucra personalidades con características heterogéneas en el proceso de vida y propicia el bienestar y sostenibilidad individual y social.

Partiendo de lo anterior, la comunicación para el desarrollo, reconoce que la protección de capital social, económico y ambiental, son aspectos necesarios para lograr un desarrollo comúnmente productivo.

Frente a este escenario, gestionar estrategias de comunicación para el desarrollo, con el propósito de obtener como resultado una sociedad saludable, es un reto que debe asumirse desde cualquier individuo, sector, comunidad, ya sea a nivel local, regional o nacional. En relación con esto, para el caso latinoamericano resulta casi inabordable, dado a que la dinámica presente en esta región es muy cambiante y por consiguiente, la gestión en función de lo saludable, se limita para suplir la necesidades básicas insatisfechas de la población vulnerable ya que sencillamente falta un modelo de planeación no solo social sino, político y económico en constante transformación. De ese modo, se prevé que la comunicación como mediadora del desarrollo, debe ser de obligatorio reconocimiento por el Estado como responsable de la gestión y ejecución de políticas públicas. (Martínez, 2012)

En esta dirección, la conciencia pública, la educación y la capacitación son claves para llevar a la sociedad hacia la sostenibilidad; pero se necesita reconocer entonces que las tendencias de desarrollo económico actuales no son sostenibles, es por eso, que la educación es una herramienta esencial para el logro de la sostenibilidad social.

En función de lo anterior, Macedo B. \& Salgado C. (2007) manifiestan que se requieren cambios urgentes y sostenidos en el tiempo que permitan el cuidado de la vida en toda su diversidad, lo que incluye la protección y la restauración de los ecosistemas; la consolidación de la democracia; la construcción de la paz; la estabilidad dentro y entre los países; la creación de sociedades más justas donde el conocimiento se distribuya equitativamente; se respete y donde se promueva la diversidad en todas sus manifestaciones.

Frente estas aseveraciones, es el momento de que las miles de intenciones y propuestas de desarrollo miren a la Educación y a la Comunicación como apuestas necesarias para poder entender desde lo particular las necesidades y oportunidades a las cuales atiende cada entorno. Estas deben mirarse desde una perspectiva conjunta para facilitar las acciones en pro del desarrollo al cual dicho entorno desea llegar.

Por otro lado, es pertinente mencionar a Freire, quien expresa que la educación a su vez es un acto político, un acto de conocimiento y un acto creador, entonces no se puede si no hacerse el mismo camino que la Comunicación en el proceso del cambio social. Gumucio, (2007).

En relación con lo anterior, la Comunicación para el Desarrollo hace una clara distinción entre información y comunicación, porque la información no involucra a las personas, en cambio, la comunicación sí lo hace. Esta dirige su accionar hacia la gestión, divulgación y producción de contenidos con el fin de transformar la calidad de vida, los malos hábitos de higiene personal y ambiental en los diferentes grupos, comunidades o sectores vulnerables. (Benavides, 2009) 
En síntesis, todo esto implica la transformación de dinámicas y comportamientos en los distintos grupos, comunidades o sectores, lo que sin duda incide en la sostenibilidad del desarrollo humano. Es aquí donde la comunicación como agente movilizador emprende estrategias de persuasión, enseñanza-aprendizaje, promoción y prevención de riesgos y factores socio-ambientales.

En últimas, la importancia que genera la Comunicación para el Desarrollo dentro de una comunidad o espacio determinado es indiscutible cuando se reconoce que es necesario cambiar el modelo vigente hegemónico para que no se profundice entre otros, la crisis ambiental que nos afecta a todos.

Respecto a la Comunicación para el Cambio Social nace según Gumucio, (2004) como respuesta a la indiferencia y al olvido, rescatando lo más valioso del pensamiento humanista que enriquece la teoría de la comunicación: la propuesta dialógica, la suma de experiencias participativas y la voluntad de incidir en todos los niveles de la sociedad. A su vez, Gumucio rescata que el proceso de formulación de la Comunicación para el Cambio Social comenzó a gestarse en abril de 1997, en una reunión convocada por la Fundación Rockefeller, en Italia. En esta primera etapa se definió progresivamente el concepto de Comunicación para el Cambio Social como un proceso de diálogo privado y público, a través del cual los participantes deciden quienes son, qué quieren y cómo pueden obtenerlo.

El enfoque inicial subraya la necesidad de cambiar los términos hasta entonces vigentes en el desarrollo y en la comunicación. Se plantea que las comunidades deben ser actores centrales de su propio desarrollo, que la comunicación no debe persuadir sino facilitar el diálogo y que no debe centrarse en los comportamientos individuales sino en las normas sociales, las políticas y la cultura. En una segunda etapa, apoyada también por la Fundación Rockefeller, se vio la necesidad de vincular el proceso de reflexión a experiencias concretas, en el sentido de que si bien el cuerpo teórico no estaba totalmente definido, la realidad mostraba, en cambio una variedad de experiencias.

En consonancia, Sosa, (2010) nos indica que la Comunicación para el Cambio Social aparece como un paradigma donde se rescata y profundiza el camino recorrido por la comunicación para el desarrollo y por la comunicación participativa incorporando nociones innovadoras y progresistas de los modelos de modernización. Cuestionando además, el concepto de un desarrollo que no cuente con la participación de los sectores directamente afectados y promueve una comunicación que haga efectiva la participación comunitaria particularmente de los sectores más pobres y aislados.

Esta aproximación enfatiza la necesidad de propiciar mayores espacios de empoderamiento, toma de decisión por parte de grupos o comunidades con las que se trabaja y sostenibilidad de los procesos. Bajo esto, Rodríguez, Obregón \&, Vega, (2002) plantean que la comunicación para el cambio social se define como procesos de diálogo público y privado a través del cual las personas definen quienes son, qué es lo que quieren y cómo lo pueden obtener.

Por otro lado, sus objetivos o directrices apuntan a que la gente sea actante de los mismos procesos; que pueda proponerlos, formularlos, gestionarlos, evaluarlos y solucionarlos. Es además, un abordaje al área de las comunicaciones que busca favorecer un desarrollo social basado en principios de justicia, equidad, respeto y diversidad. 
Es la conceptualización de la comunicación como un proceso que dirige sus acciones desde el dialogo y la participación de la ciudadanía; generando estrategias para ayudar a que las personas logren un poder que les permita participar activamente en la solución de sus necesidades y es la comunicación la que figura como centro en este proceso de desarrollo.

La Comunicación para el Cambio Social ha logrado acaparar la atención de los estudiosos del campo de la comunicación en salud, especialmente en África, Asia y América Latina. Sus postulados trastocan los planteamientos hechos por varios modelos y teorías del campo de la comunicación en salud, los cuales en su mayoría atienden el carácter individual de un sujeto como parte de las características principales de estos enunciados. En este contexto, para Ríos, (2011) el paradigma de la Comunicación para el Cambio Social es sin lugar a duda uno de los fenómenos que ha logrado acaparar la atención de estudiosos de la comunicación en salud en los últimos años, dado a la importancia que le brinda a la participación comunitaria en todos los asuntos que rodean el desarrollo e implementación de un esfuerzo que promueve mejores estilos de vida en la población. Este modelo establece una relación directa entre el proceso de comunicación y el desarrollo de los pueblos. Además, trastoca la pasividad que se le atribuía al receptor en un proceso de comunicación tradicional o unilineal (Fuente- Mensaje- Canal- Receptor).

Siguiendo a Ríos, este autor enfatiza en que es necesario hacer un análisis ponderado de los principales conceptos y modelos teóricos predominantes en el campo de la comunicación en salud. Además, es pertinente examinar de qué forma el innovador paradigma de la Comunicación para el Cambio Social se convierte en una aportación significativa para el entendimiento de los procesos que pretenden cambios de conducta en la población. (Ríos, 2011)

En consecuencia, el cambio social ejercido desde la comunicación, es un estado que permite a la sociedad progresar desde lo cultural hasta lo económico facilitándose su sostenimiento y el de las generaciones futuras. Mirado así, los componentes principales del desarrollo serian: desarrollo local, sostenibilidad y participación.

En última instancia desde el paradigma de la Comunicación para el Cambio Social, Mosquera, (2003) nos describe a este como un proceso donde el "diálogo de la comunidad" y la "acción colectiva" trabajan en conjunto para producir cambios sociales en una comunidad que mejoren el estado de salud y de bienestar de todos sus miembros.

El cambio social implica la participación de la comunidad en todos los procesos concernientes a la implementación de proyectos para la salud. Asimismo, la horizontalidad de la comunicación ejerce un papel fundamental para que la población adopte como suyos, los métodos y los estilos de vida necesarios para su sostenibilidad.

Estos procesos deben ser orientados a facilitar los cambios sociales a nivel de comunidades, Estados a nivel internacional. Procesos como estos, aunque pretendan y sustenten su accionar desde lo dialógico, utilizan además medios de comunicación de toda índole, ya sean tradicionales o modernos, alternativos o participativos o de masas.

Lo más respetable en la Comunicación para el Cambio Social es la posibilidad de que sean las personas de la comunidad las que formulen, evalúen, y propongan sus propios procesos de o para el cambio. 
En lo referente a la Comunicación en Salud, Atoche, (2003) expresa que al término Promoción para la Salud se le sumó Educación para la Salud y por ultimo Comunicación para la Salud (OPS/OMS). En ese sentido, desde 1993 con la celebración del 39 Consejo Directivo de la OPS se aprobó la resolución "Promoción de la salud en las Américas", por la cual "insta a los gobiernos miembros a que incluyan, como instrumentos claves en los programas de salud de la comunidad, campañas de comunicación social y de educación, promoviendo la responsabilidad de la población".

Esto se reafirmó en la Declaración de Yakarta sobre Promoción de la Salud en el siglo XXI, adoptada en la Conferencia Internacional sobre la Promoción de la Salud, celebrada en julio de 1997, la cual indica que el acceso a los medios de información y la tecnología de las comunicaciones es importante para adelantar la promoción de la salud.

Desde esta perspectiva, las definiciones de Comunicación en Salud han evolucionado, incluyendo cambios sustanciales en la planeación y la conceptualización utilizada a su desarrollo. (Atoche, 2003)

Según Healthy People, OMS (2010, Volumen I), la comunicación en salud abarca el estudio y el uso de estrategias de comunicación para informar e influenciar decisiones individuales y comunitarias que mejoren la salud. Este tipo se comunicación es reconocida como elemento necesario en los esfuerzos para mejorar la salud pública y personal.

Por su parte, Mosquera, (2003) manifiesta que la comunicación en salud puede contribuir en todos los aspectos de la prevención de la enfermedad incluyendo las relaciones médico-paciente, la adherencia del individuo a recomendaciones clínicas y regímenes terapéuticos, la construcción de mensajes y campañas de salud pública en conjunto con la diseminación de información concerniente a riesgos para los individuos y las poblaciones, o comunicación preventiva.

Tufte, (2004) a su vez, nos enmarca en que es en el campo de la comunicación para la salud el que ha conducido las estrategias de comunicación a su máximo nivel.

A su modo, Ríos, (2011) señala que la comunicación en salud ha sido interpretada o definida a través de los años como el estudio de la naturaleza, alcance y función, así como los medios por los cuales los tópicos de salud alcanzan y afectan las audiencias apropiadas. Las áreas de estudio incluyen: métodos de formulación de mensajes, estrategias de implantación y técnicas de avalúo.

Bajo lo anterior, Ratzan, (1994) citado por Ríos, (2011) menciona que el Centro de Control de Enfermedades (CDC) en los EEUU define la comunicación en salud como -el arte y la expresión de mensajes y estrategias, basado en la investigación del consumidor, para promover la salud de los individuos y las comunidades.

En el contexto académico, la Comunicación en Salud se ha definido como el arte y la técnica de informar, influir y motivar audiencias a nivel individual, institucional y público acerca de asuntos de salud importantes. Su alcance incluye prevención de enfermedad, política de cuidado de salud, así como el aumento de la calidad de vida y salud de los individuos dentro de la comunidad. (Ratzan, citado por 1994 Ríos, 2011)

Ríos, (2011) enfatiza además, en que uno de los más recientes enfoques propuestos para catalogar el proceso de comunicación en salud, y que es defendido por autores como Gumucio, quien expone la necesidad de una comunicación para la salud comunitaria, está basado en el diálogo.

De acuerdo con este nuevo paradigma, se entiende que las comunidades deben ser protagonistas y gestoras de los cambios que las afectan. Debieran del mismo modo ser 
responsables del manejo de su salud y en consecuencia, de su propia comunicación. (Gumucio, 2001 citado Ríos, 2011).

Siguiendo este nuevo enfoque, y catalogado como comunicación alternativa- representa entonces, un reto para las autoridades gubernamentales, y privadas encomendadas al desarrollo de esfuerzos que promueven cambios de conductas, y mejores estilos de vida en la población, ya que trastoca los enfoques tradicionales de comunicación en salud predominantes por las últimas décadas.

En cuanto a participación comunitaria en Salud, Gumucio, (2001) hace una comparación entre los programas de salud y los programas de comunicación. El diagnóstico, la planificación y la ejecución en los programas de salud suelen ser procesos unidireccionales y verticales. En un extremo están las organizaciones y los sistemas que generan las acciones preventivas o correctivas, y en otro extremo los "receptores", los "destinatarios" de esas acciones. Si trasladamos esto al terreno comunicacional, constatamos que hay algo que no anda bien: tenemos un polo generador de decisiones o mensajes, y por otra parte un polo receptor, aparentemente pasivo.

En síntesis, Gumucio acentúa en que ningún programa de salud que pretenda abarcar a sectores sociales cada vez más amplios puede siquiera pensarse al margen de la comunicación. La comunicación es imprescindible para ganarle tiempo al tiempo, para recuperar el tiempo perdido, para resolver a contrarreloj la gravísima situación que se vive -y se muere- en el terreno de la salud materno-infantil, de la salud rural, de la salud en las poblaciones urbanas marginales.

Desde esa perspectiva, la comunicación para la salud deja de ser una responsabilidad concentrada en las instancias del gobierno o de los organismos internacionales especializados. Se enriquece la pluri-direccionalidad de la comunicación, y naturalmente de los contenidos, mediante la participación de organizaciones sindicales, grupos de base, instituciones no-gubernamentales y las propias comunidades urbanas y rurales. (Gumucio, 2001).

Luego de una revisión se presentan a continuación, los conceptos básicos de las teorías y modelos de comunicación en salud más abordados por investigadores, teóricos y expertos en comunicación en salud, los cuales enfatizan en el cambio de comportamiento, y asimismo serán empleadas para contrastar si la estructura de dichos Planes se ajustan a algún modelo o teoría de comunicación y posteriormente identificar qué tanto de alejan o se a cercan al componente de participación desde el enfoque de Comunicación para el cambio social. 
Cuadro No. 1 Síntesis Modelos y Teorías de Comunicación en Salud

\begin{tabular}{|c|c|}
\hline TEORIA/MODELO & SINTESIS \\
\hline $\begin{array}{l}\text { Teoría de Acción } \\
\text { Racionada propuesto por } \\
\text { Fishbein y Ajzen }\end{array}$ & $\begin{array}{l}\text { Plantea que existe un factor determinante del comportamiento y } \\
\text { es la intención de la persona lo realice: } \\
\text { A) la actitud de la persona hacia el desarrollo del } \\
\text { comportamiento. Y B) la percepción de las personas sobre la } \\
\text { presión social ejercida sobre él o ella a realizar el } \\
\text { comportamiento. }\end{array}$ \\
\hline $\begin{array}{l}\text { Teoría de Aprendizaje } \\
\text { Social Propuesta por } \\
\text { Albert Bandura }\end{array}$ & $\begin{array}{l}\text { Los cambios de comportamiento son el resultado de la } \\
\text { interacción de la persona con el medio ambiente, el entorno en } \\
\text { general y el dialogo contractivo con el mismo. }\end{array}$ \\
\hline $\begin{array}{l}\text { Modelo de Creencias de } \\
\text { la Salud por Rosenstock }\end{array}$ & $\begin{array}{l}\text { Dos factores primordiales influyen en que una persona adopte } \\
\text { una acción recomendada. } \\
\text { Primero, debe sentirse personalmente amenazada por la } \\
\text { enfermedad debe sentirse personalmente susceptible a la } \\
\text { enfermedad con serias o severas consecuencias. } \\
\text { Segundo, debe creer que los beneficios de tomar las acciones } \\
\text { preventivas son mayores que las barreras (y/o costos) a la } \\
\text { acción preventiva. }\end{array}$ \\
\hline $\begin{array}{l}\text { Modelo Transteorico o } \\
\text { de Cambio deProchaska }\end{array}$ & $\begin{array}{l}\text { Las personas pueden tardarse un gran periodo de tiempo en } \\
\text { moverse a la siguiente etapa del Modelo de Cambio definida } \\
\text { como determinación. } \\
\text { En esta etapa las personas combinan la intención con los } \\
\text { criterios de conducta que podrían adoptar. }\end{array}$ \\
\hline $\begin{array}{l}\text { Modelo de Comunicación } \\
\text { para el Cambio Social } \\
\text { propuesto por Gumucio } \\
\text { entre otros. }\end{array}$ & $\begin{array}{l}\text { Describe un proceso donde el "diálogo de la comunidad" y la } \\
\text { "acción colectiva" trabajan en conjunto para producir cambios } \\
\text { sociales en una comunidad que mejoren el estado de salud y de } \\
\text { bienestar de todos sus miembros. }\end{array}$ \\
\hline $\begin{array}{l}\text { Modelo Socio-ecológico } \\
\text { propuesto por Hernán San } \\
\text { Martin. }\end{array}$ & $\begin{array}{l}\text { La salud de los individuos y de la población organizada depende } \\
\text { de múltiples factores, que también constituyen un sistema: el } \\
\text { sistema biológico-social de la salud y la enfermedad. } \\
\text { El "sector salud" está considerado como un subsistema con su } \\
\text { organización y sus procesos internos. Están definidas sus } \\
\text { conexiones con el sistema social. }\end{array}$ \\
\hline
\end{tabular}

Fuente: Martínez, (2013) 


\section{CONCLUSIONES Y RECOMEDACIONES}

El tema de Comunicación en Salud que apropia aspectos del nuevo concepto de salud Pública, permite que directamente se hable de desarrollo humano, donde es necesario dialogar constructivamente con las comunidades y reconocer de acuerdo a sus diferencias, a qué necesidades responden.

Lo interesante de la Comunicación en Salud, es que sean esas poblaciones las que manifiesten sus problemas, formulen y gestionen las posibles soluciones, asimismo las evalúen y logren un verdadero cambio de comportamiento; y para ello se requiere que el componente de Comunicación para el Cambio Social actué como agente dinamizador.

Se concluye que así como la Comunicación para el Desarrollo facilita la identificación de necesidades a través del diseño y producción de mensajes para poblaciones con características homogéneas, y la Comunicación para el Cambio Social es un abordaje al área de las comunicaciones que dirige sus acciones desde el dialogo y la participación de la ciudadanía, la comunicación en Salud irrumpe como un llamado a que el proceso de salud-enfermedad sea más dialógico, activo, fortalezca la resiliencia y que desde este mismo proceso todos nos hagamos responsables por nuestra comunicación y las alternativas de cambio. Y, eso contribuye sí que a mejorar los estilos de vida saludables.

Se concluye exponencialmente, que la salud es un proceso que abarca dimensiones como el propio desarrollo. Asimismo, la Comunicación en Salud interviene en diversas etapas del desarrollo del individuo, lo que genera opciones y respuestas al proceso de salud-enfermedad.

Se recomienda que nivel estatal se revise la política pública concretamente y se identifique si realmente se están formulando acciones que influyan positiva y eficientemente en las comunidades y si éstas inciden en su cambio de comportamiento en función de generar estilos de vida saludables. ¿Qué está pasando con la comunicación?

En últimas, se recomienda que desde la Comunicación en Salud, se propenda por la formulación y ejecución de estrategias de comunicación con enfoque diferenciado y que respondan a necesidades concretas de las comunidades o poblaciones; éstas, a su vez conllevarían a generar el cambio de comportamiento en los individuos a fin de lograr estilos de vida saludables ya que se enfatiza en un cambio estructural, de este modo, se lograría transitar hacia el autodesarrollo. 


\section{REFERENCIAS}

1. Ascroft y Masilela, (1989) en Snirivas R., Melkote. Theories of Development Communication. Bowling Green State University. Págs. 129- 144.

2. Atoche M. (2003). Comunicación y Salud: Periodismo institucional y de difusión en la oficina de prensa del Ministerio de Salud. Tesis Licenciatura; publicada, Universidad de Lima, Lima, Perú.

3. Beltrán, L. (1997). Universidad y Comunicación para la salud, acciones y reflexiones sobre comunicación y salud. Documento elaborado en la Primera Reunión Internacional sobre Comunicación y Salud. Lima.

4. Benavides P. (2009). Manifiesto para la Comunicación Sostenible. [Consultado 30 agosto 2011] Disponible en:

http://www.elblogalternativo.com/2009/07/21/manifiesto-para-la-comunicacionsostenible-de-pablo-benavides/

5. Blumer y Catz, (1974) en Snirivas R., Melkote. Theories of Development Communication. Bowling Green State University.Págs. 129- 144.

6. Diaz B, (1989) en Snirivas R., Melkote. Theories of Development Communication. Bowling Green State University.Págs. 129- 144.

7. Fawcett, (1984) en SNIRIVAS R., Melkote. Theories of Development Communication. Bowling Green State University.Págs. 129- 144.

8. Gumucio, A. (2001). Haciendo Olas historias de comunicación participativa para el cambio social. Fundación Rockefeller. New York.

9. (2004). El cuarto Mosquetero: Comunicación para el cambio social. Investigación y Desarrollo. Vol.12, 1, pp. 2-2. Universidad del Norte.

10. (2007). Comunicación para el cambio social: clave del desarrollo participativo. Signo y Pensamiento, vol. 30 № 58. Universidad Pontificia Javeriana.

11. (2001). Comunicación para la Salud: el Reto de la Participación. [Consultado agosto 11 de 2012] en Portal COMMINIT. Disponible en: http://www.comminit.com/en/node/150405

12. Macedo B, Salgado, C. (2011) Educación ambiental y educación para el desarrollo sostenible en América Latina-OREALC/UNESCO Santiago (Oficina Regional de Educación de la UNESCO para América Latina y el Caribe). 1:29- 37.

13. Martínez C. (2012). Comunicación para el desarrollo. Una mirada a la relación entre salud, productividad, sostenibilidad y sustentabilidad social. En: Memorias XIV Encuentro Latinoamericano de Facultades de Comunicación, FELAFACS. Universidad de Lima, Perú.

14. Martínez, C. (2012). Medio Ambiente y Desarrollo. Una Mirada a la relación entre productividad, sostenibilidad y sustentabilidad social. En R. Román, A. Vidal, \& J. Caro, Imperios, Mercados y Multiculturalidad en el Caribe: Memorias II Congreso Internacional De Estudios Caribeños (pp 348-362). San Andrés Isla: Universidad Nacional de Colombia, Sede Caribe.

15. Martínez, C. (2013) Una mirada desde la Comunicación en Salud a los Planes de Promoción de Salud en Suramérica. Casos: Bolivia, Chile, Colombia. Tesis inédita Pregrado Profesional en Dirección y Producción de Radio y Televisión. Universidad Autónoma del Caribe.

16. Mosquera, M. (2003). Comunicación en Salud: Conceptos, Teorías y Experiencias. [Consultado mayo 20 de2012] en Portal COMMINIT. Disponible en: http://www.comminit.com/la/lasth/sld-2915.html

17. Rahman, (1991) en Snirivas R., Melkote. Theories of Development Communication. Bowling Green State University.Págs. 129- 144

18. Rappaport, (1987) en Snirivas R., Melkote. Theories of Development Communication. Bowling Green State University.Págs. 129- 144. 
19. Ríos, I. (2011). Comunicación en Salud: conceptos y modelos teóricos. Perspectivas de Comunicación. Vol. 4, № 1, Universidad de la Frontera.

20. Rodríguez C., Obregón R. y Vega J. (2002). Estrategias de Comunicación para el Cambio Social- Ecuador Impresiones MYLQuito. Pág. 24 -10- 16, 17, 23.

21. Sen, G. (2007). El Empoderamiento como un enfoque a la pobreza. Tomado de Comunicación, Participación y Cambio Social. Pág 29, 31. Universidad del Norte Barranquilla.

22. Shaw y Mccombs, (1974) en Snirivas R., Melkote. Theories of Development Communication. Bowling Green State University.Págs. 129- 144.

23. Singhal y Rogers, (1999) en Snirivas R., Melkote. Theories of Development Communication. Bowling Green State University.Págs. 129- 144.

24. Sosa, M. (2010). Recomendaciones estratégicas en Comunicación participativa para responder a la problemática en Salud Sexual, Reproductiva y Preventiva para los jóvenes aprendices del centro de comercio y servicios del SENA Regional Atlántico, Barranquilla. Tesis inédita Maestría en Comunicación. Universidad del Norte.

25. Tufte T., (2004). Eduentretenimiento en la comunicación para el vih/sida. Más allá del mercadeo, hacia el empoderamiento. Investigación y desarrollo vol $12, \mathrm{n}^{\circ} 1$ (2004) p ágs 24-43. Universidad del Norte.

26. OMS (2010). Healthy People, Volumen I. Disponible en: http://www.healthypeople.gov/2020/default.aspx

\section{Cómo citar el artículo}

Martínez Beleño, C. A.; Sosa Gómez, M. S. (2016). Aportaciones y diferencias entre comunicación en salud, comunicación para el desarrollo y para el cambio social.

Revista de Comunicación y Salud, Vol. 6, pp. 71-82.

DOI: http://doi.org/10.35669/revistadecomunicacionysalud.2016.6.71-82 